\title{
Magnetic Cataclysmic Variables: a Summary
}

\author{
Jean-Pierre Lasota \\ UMR 7095 du CNRS, Institut d'Astrophysique de Paris, \\ 98bis Boulevard Arago, 75014 Paris, France
}

\begin{abstract}
This is a short summary of the main problems presented and discussed at the Cape Town conference.
\end{abstract}

\section{Introduction}

This has been the third conference about Magnetic Cataclysmic Variables (MCVs). The first conference took place in South Africa and after seven years we had the pleasure of returning to this beautiful and friendly country. It was a special pleasure to see that South Africa is encouraging astronomy, as the SALT and other projects show [Buckley].

What are MCVs? Why a conference about just magnetic CVs and not their non-magnetic cousins? In fact, during the conference there was an (unfortunately not too successful) attempt to tackle the first question. (Presumably the participants in this conference believed in the utility of MCVs conferences.) But this attempt (a short discussion) was concerned more with the classification of MCVs then with the definition itself. For the purpose of this summary I will define MCVs as cataclysmic variables in which the magnetic field of the white dwarf determines one of the fundamental properties of the system. This is obviously the case of "standard" MCVs such as AM Her stars and intermediate polars (IPs) but other systems believed to belong to other CV classes might at some times be observed as MCVs: for example, they could reveal their magnetic character only at low mass-transfer rates. This is not merely playing with words: such a broad definition of MCVs might help us to understand the secular evolution of CVs.

In general MCVs, especially AM Her stars not polluted by accretion discs, provide a unique view of the interaction between the binary components, of the variation of the mass-transfer rate and of some accretion phenomena such as column accretion. The wealth of effects related to the magnetic character of CVs and the fascinating physics they involve certainly deserve specialized conferences. Especially since there are still a lot of things to understand. A young participant told me: "I came here to understand and obtain answers, but instead I got mostly questions".

\section{Population and evolution}

The highlight of the conference was the confirmation of existence of a new class of MCVs [Tovmassian, Schmidt, Szkody]: the very low accretion rate polars 
(VLARPs - adding a "V" to LARP stresses that one describes systems with accretion rates lower than those found during "usual" low states of polars). These are MCVs with magnetic field typical of AM Her stars but accreting at rates $\sim 10^{-12}-10^{-14} \mathrm{M}_{\odot} \mathrm{y}^{-1}$, i.e. at rates lower than implied by gravitational radiation for a semi-detached systems. Known before (Schwope et al. 2002), they are now being discovered in large quantities mainly by the Sloan Digital Sky Survey. Low accretion rates imply low white-dwarf temperatures so it is not surprising that the coolest white dwarfs among CVs are found in MCVs [Sion]. (The hottest is a dwarf nova, which shows that disc irradiation by hot white dwarfs does not prevent instabilities and therefore imply that VY Scl are magnetized [Lasota]).

Several of VLRAPs have orbital periods in the $\sim 2-3 \mathrm{~h}$ period range. Since out of 51 systems in the period range 25 are magnetic one can have reasonable doubt about the existence of a "gap" for MCVs. The certitude in this matter will be reached when the relation between polars and intermediate polars [Norton] and the problem of AM Her star progenitors (King \& Lasota 1991; Patterson et al. 2002) are finally solved. It is tempting to assume that VLRAPs are in the gap, in the sense that they would be detached as the result of a secular reduction of the magnetic braking, however, the presence of a VLRAP at $4.06 \mathrm{hr}$ makes this hypothesis not very attractive. Are VLRAPs a different population of MCVs [Schmidt] ? Or are they subject to much longer "low states" than the "usual" polars. The answer to that question is obviously related to the problem of the origin of mass-transfer rate variations in MCVs and "non-magnetic" systems [Hessman].

The standard model of CV evolution has been recently challenged from both observational and theoretical points of view (e.g. Baraffe \& Kolb 2000; Andronov et al. 2003) but despite of that the "traditional" framework is still in use (sometimes slightly modified as in Webbink \& Wickramasinghe 2002). In any case such essential parameters of MCVs as masses (of both components) and magnetic fields can be seriously affected during some episodes of binary evolution such as the thermal timescale mass transfer phase [Schenker] and by magnetic field re-emergence [Cummings]. The origin of MCVs' magnetic fields is still an open question [Shylaja].

The UCT CCD CV Survey is finding MCVs among classical novae [Woudt]. The relations between the two classes of objects still awaits a satisfactory analysis. The same is true of so called "peculiar" MCVs showing unusual CNO line ratios implying non-solar composition of the accreting matter[Mouchet, BonnetBidaud]. Clearly, close binary evolution still has a bright future.

\section{The structure of magnetized accretion flows}

Some VLRAPs might not be accreting at all [Ramsay], but when they accrete their accretion mode can be described by the bombardment solution (Woelk \& Beuermann 1992). The bombardment solution uses a kinetic description of the flow. The fluid (or one- or two-fluid) description of the accretion plasma (appropriate for higher $\dot{M}$ ) is an extremely complicated radiation-hydrodynamic problem in which, as usual, the role of collective plasma effects is difficult to 
take into acount [Beuermann; Serber]. At least modelling of the magnetic field structure gives interesting results [Reinsch].

Important diagnostics of accretion flows is provided by spectroscopy in various wavelengths [Mauche, Mukai, Schlegel, Singh, Barret]. It appears that both in AM Her stars and IPs accretion flows are hot and dense [Burwitz]. Many questions are still to be answered: what is the validity of the cooling-flow model applied to MCVs? Does the accretion flow pattern changes in hours? Are velocity shifts really observed?

Surprisingly (for a theorist at least) unexpected questions were asked, e.g.: "where is the white dwarf in UZ For?" Apparently, such problems are (luckily) just the result of using softwares unadapted to new modern timing standards [Cropper].

The "standard" accretion-column solution, first published by King \& Lasota (1979), could not, as noticed by these authors, reproduce soft $X$-ray excess observed in some, not very numerous at that time, systems. Now this model appears to suffer from the opposite problem: the absence of a "distinct" soft Xrays (from a least one pole) in 6 polars observed by XMM - Newton [Ramsay]. The strength of the soft X-ray component might depend on the strength of the magnetic field [Beuermann)] but probably more careful modelling of reprocessed spectra is also required [Ramsay].

The nature of the so-called accretion events occurring usually (or at least usually observed) during low states requires explanation. They could be due to a companion coronal mass ejection or to instabilities in the accretion flow [Schwope]. It worth reminding that similar accretion events are seen in quiescent dwarf novae and soft X-ray transients.

Another problem presumably related to the magnetic structure of MCVs is their radio emission. Few are radio-loud [Venter], the majority is radio-quiet. Why?

\section{The "blob paradigm", weak fields and related systems}

The blob picture of accretion flows on to MCVs advocated mainly by Andrew King and collaborators (e.g. Hameury, King \& Lasota 1986) seems to be confirmed by observations [Gomer] and is widely used in theoretical models. The clever but rather simple description of diamagnetic blobs proposed by King (1993) is being at last refined and one finds now things like "genetically modified fire-flies" used in stream-eclipse mapping [Bridge]. The blob physics is given more attention [Halevin] and flaring in general could be attributed to "fireballs" [Pearson].

It is not clear yet what is the relation of ultra-short $(<10 \mathrm{~min})$ binaries to polars since they could be electric rather magnetic stars [Cropper, Israel]. Whatever their electromagnetic nature (if any) they should be rather noisy sources of gravitational radiation easily detected by LISA.

Finally, it is now clear that the fascinating correlation observed between pairs of quasi-periodic oscillations (QPO) in Low Mass X-ray Binaries extends down to cataclysmic variables covering now six order of magnitude [Warner]. In CVs correlated QPOs are observed both in optical and in soft X-rays (Mauche 
2003). The fact that also CVs are members of the club shows that the correlation is not, as has been generally believed, due to some effects of General Relativity.

\section{Future directions}

In observations the future belongs to large surveys like the SSDS (especially for finding very weak sources) and observations by instruments such as FUSE and XMM OM. High resolution spectroscopy should play a crucial role. As usual multi-wavelength observations are highly recommended.

As mentioned above several fundamental questions still await answers. Is the MCV evolution different from that of non-magnetic CVs? Do very low $-\dot{M}$ MCVs form a separate population? Progress is needed in the understanding of the structure of the magnetic fields in MCVs and the plasma physics (including radiation transfer) of accretion flows. It is, however, difficult to be optimistic about the last point considering the usual horrors of plasma physics involved. It is a personal view that looking for polarized radiation periodic pulsations in VY Scl stars and other Nova-Like binaries should confirm the magnetic nature of many of these systems.

One can hope that the 2004 conference "The astrophysics of cataclysmic variables and related objects" in Strasbourg (France) will bring some answers to the many questions asked at the Cape Town meeting.

\section{References}

Andronov, N., Pinsonneault, M., \& Sills, A. 2003, ApJ, 582, 358

Baraffe, I., \& Kolb, U. 2000, MNRAS, 318, 354

Hameury, J.-M., King, A.R., \& Lasota, J.-P. 1986, MNRAS, 218, 695

King, A.R. 1993, MNRAS, 261, 144

King, A.R., \& Lasota, J.-P. 1979, MNRAS, 188, 653

King, A.R., \& Lasota, J.-P. 1991, ApJ, 378, 674

Mauche, C.W. 2002, ApJ, 580, 423

Patterson, J., Fenton, W.H., Thorstensen, J.R. (+15 co-authors) 2002, PASP, 114,1364

Schwope, A.D., Brunner, H., Hambaryan, V., \& Schwarz, R. 2002, ASP Conf. Ser. 261: The Physics of Cataclysmic Variables and Related Objects, 102

Webbink, R.F. \& Wickramasinghe, D.T. 2002, MNRAS, 335, 1

Woelk, U. \& Beuermann, K. 1992, A\&A, 256, 498 